\title{
Avaliação da perviedade precoce das fístulas arteriovenosas para hemodiálise
}

\author{
Evaluation of early patency of the arteriovenous fistulas for hemodialysis
}

Milton Alves das Neves Junior', Rafael Couto Melo², Catarina Coelho de Almeida ${ }^{3}$, Allison Roxo Fernandes Alexandre Petnys ${ }^{5}$, Maria Lucia Sayuri Iwasaki ${ }^{6}$, Edgar Raboni ${ }^{7}$

\section{Resumo}

Contexto: A insuficiência renal crônica é uma doença de alta prevalência e morbidade, o que determina queda da qualidade de vida. Pacientes em hemodiálise necessitam de um acesso vascular que permita a conexão da circulação do paciente ao circuito externo de hemodiálise. Dentre os acessos disponíveis, as fístulas arteriovenosas (FAV) são as que mais se aproximam do acesso ideal.

Objetivo: Avaliar a perviedade precoce das FAV, identificando os fatores relacionados ao insucesso destas.

Métodos: Foram acompanhados todos os pacientes submetidos à confecção das FAV no Hospital do Servidor Público Municipal de São Paulo, no período de agosto de 2008 a janeiro de 2009, avaliando-se a perviedade destas no 1, $10^{\circ}$ e $30^{\circ}$ pós-operatório. Foram realizadas $31 \mathrm{FAV}$ no período, apresentando-se média de idade de 63,06 anos, sendo 18 pacientes do sexo masculino e 13 do feminino.

Resultados: Vinte e seis FAV foram distais, todas radiocefálicas; quatro foram proximais, das quais duas braquiocefálicas e duas braquiobasílicas superficializadas; uma FAV confeccionada com alça de politetrafluoretileno (PTFE) fêmoro-femoral esquerda. A taxa de perviedade no primeiro mês foi de $71 \%$ dos casos. $O$ uso de cateteres venosos centrais apresentou-se como fator de risco para oclusão da FAV ( $p=0,01$ ). As FAV continuam sendo o acesso vascular para hemodiálise mais aceito e mais seguro. A indicação precoce para confecção das FAV é de fundamental importância, evitando-se, assim, o uso de cateteres e suas complicações.

Conclusões: A perviedade precoce encontrada neste estudo é semelhante à da literatura, e o uso prévio de cateteres é o fator de risco mais significativo para oclusão precoce desta.

Palavras-chave: Fístula arteriovenosa, diálise renal, desobstrução vascular

\begin{abstract}
Background: Chronic renal failure is a disease of high prevalence and high morbidity, which impairs the patients' quality of life. Patients on hemodialysis need a vascular access for connection with the hemodialysis equipment. Arteriovenous fistulas (AVF) are the best option for this purpose.

Objective: To evaluate the early patency of AVFs and to identify the causes of their failure.

Methods: All patients AVF underwent operations for dialysis from August, 2008 to January, 2009. The AVF patency was evaluated on the $1^{\text {st }}, 10^{\text {th }}$ and $30^{\text {th }}$ postoperative days. Thirty-one operations for AVF were performed in our hospital in the period; 18 patients were males and the mean age was 63.03 years.

Results: Twenty-six AVF were distal, all radial-cephalic; four were proximal, out of which two were brachiocephalic and two were brachiobasilic; one AVF was a femoral-femoral loop PTFE graft. The patency rate in the first month was of $71 \%$. The use of central venous catheters was a risk factor for AVF occlusion $(p=0.01)$. AVF remains the most accepted and safe vascular access. Early performance of the AVF is very important to avoid the complications resulting from long-term use of central venous catheters.

Conclusions: Data regarding early patency found in this study were similar to those found in literature, and the use of central venous catheters before performing AVF is an important risk factor for occlusion.
\end{abstract}

Keywords: Arteriovenous fistula, renal dialysis, vascular patency.

O trabalho trata-se se um estudo prospectivo não-randomizado. Mesmo assim, foi apreciado e aprovado pelo Comitê de Ética em Pesquisa da nossa instituição, conforme citado na metodologia do trabalho. Este está protocolado sob o Parecer n $174 / 2009$ deste comitê.

${ }^{1}$ Cirurgião Vascular no Hospital do Servidor Público Municipal, São Paulo (SP), Brasil.

${ }^{2}$ Cirurgião Vascular no Hospital do Servidor Público Municipal, São Paulo (SP), Brasil.

${ }^{3}$ Cirurgiã Vascular no Hospital do Servidor Público Municipal, São Paulo (SP), Brasil.

${ }^{4}$ Cirurgião Vascular no Hospital do Servidor Público Municipal, São Paulo (SP), Brasil.

${ }^{5}$ Cirurgião Vascular no Hospital do Servidor Público Municipal, São Paulo (SP), Brasil.

${ }^{6}$ Cirurgiã Vascular no Hospital do Servidor Público Municipal, São Paulo (SP), Brasil.

${ }^{7}$ Cirurgião Vascular no Hospital do Servidor Público Municipal, São Paulo (SP), Brasil.

Não foram declarados conflitos de interesse associados à publicação deste artigo.

Submetido em: 13.3.10. Aceito em: 21.3.11

J Vasc Bras. 2011;10(2):105-109 


\section{Introdução}

A insuficiência renal crônica (IRC) é uma doença de alta prevalência e morbidade, o que determina queda da qualidade de vida. Segundo dados do Sistema Único de Saúde (SUS) de 2006, o Brasil apresentava uma prevalência de 46,20 pacientes com IRC dialítica para cada 100.000 pessoas $^{1}$, sendo que a grande maioria destes encontra-se em programa de hemodiálise. Dados do Censo 2008 da Sociedade Brasileira de Nefrologia mostram que $89,4 \%$ dos pacientes dialíticos utilizam o método de hemodiálise ${ }^{2}$.

Pacientes em hemodiálise necessitam de um acesso vascular que permita a conexão da circulação do paciente ao circuito externo de hemodiálise. $\mathrm{O}$ acesso ideal é aquele capaz de fornecer um fluxo adequado, duradouro, facilmente obtido e com baixo índice de complicações (infecção, trombose etc.). Dentre os acessos disponíveis para hemodiálise, as fístulas arteriovenosas (FAV) são as que mais se aproximam do acesso ideal.

Esse tipo de acesso, desenvolvido em 1966 por Appel, e amplamente utilizado pelos nefrologistas Brescia e Cimino, foi o passo inicial para a utilização crônica da hemodiáli$\mathrm{se}^{3}$. Até então, esse método de substituição renal era utilizado para casos agudos e limitados a poucas sessões, dada a dificuldade imposta pelas repetidas punções e o rápido esgotamento dos acessos. O uso de cateteres, utilizados anteriormente nos shunts e atualmente como método de urgência ou mesmo definitivos (cateteres de longa permanência) para o acesso vascular, está relacionado com aumento da mortalidade em até 50\%, quando comparado ao uso das FAV em pacientes renais crônicos ${ }^{4}$. Além disso, esses dispositivos acabam por deteriorar o sistema venoso dos pacientes (estenoses centrais, tromboses), impedindo a confecção das FAV em alguns casos.

Sendo assim, os pacientes com IRC que necessitam de hemodiálise devem ser indicados preferencialmente à confecção da FAV, devido às várias vantagens do método em comparação com os cateteres venosos de longa permanência, conforme exposto. Um movimento norte-americano chamado fistula first ${ }^{5}$, baseado nas orientações do Kidney Disease Outcomes Quality Initiative (KDOQI) ${ }^{6}$, vem ressaltando a importância da indicação e confecção precoce da FAV, evitando a utilização dos cateteres e suas complicações. Para isso, as FAV devem ser indicadas antes mesmo de o paciente necessitar da hemodiálise, já que requerem um período para sua maturação - tempo necessário para que a veia adquira diâmetro, fluxo e trajeto adequados para suportar a diálise. As indicações para confecção da FAV são: creatinina sérica $>4 \mathrm{mg} / \mathrm{dL}$; clearance de creatinina $<25 \mathrm{~mL} /$ minutos e previsão da necessidade de diálise no próximo no $^{6}$.
A escolha do tipo de acesso a ser confeccionado é orientada por uma avaliação inicial adequada. Anamnese, incluindo a história prévia do uso de cateteres centrais e punções venosas periféricas, e o exame físico vascular detalhado são de extrema importância ${ }^{7}$. A ultrassonografia com Doppler colorido (US Doppler) pode ser útil no mapeamento venoso e arterial, orientando a confecção das FAV ${ }^{8}$.

As FAV podem ser classificadas quanto à localização e quanto ao conduto. Quanto à localização, os membros superiores são os locais de eleição e são divididos em dois grupos: distais, que incluem as FAV radiocefálicas no punho e no antebraço; proximais, que incluem as FAV braquiocefálica, braquiobasílica superficializada e braquioaxilar ou braquiobraquial em alça com prótese. Quanto ao conduto, pode ser autógeno (veias cefálica, basílica e safena) ou protético (politetrafluoretileno - PTFE ou Dacron). Um terceiro grupo que pode ser incluído seriam as FAV de exceção, como as dos membros inferiores, FAV em colar (derivação axilo-axilar), entre outras.

Vários critérios são empregados para reconhecer a maturação da FAV. Ao exame clínico, a presença de um conduto visível, com bom frêmito e facilmente puncionável, indica que a FAV é capaz de fornecer fluxo adequado para hemodiálise. À US Doppler, a maturação é definida como o diâmetro do conduto maior que $4 \mathrm{~mm}$ e fluxo maior que $400 \mathrm{~mL} /$ minutos $^{9}$.

A taxa de falha precoce encontrada na literatura varia de 10 a $27 \%{ }^{10}$. As principais causas para o não desenvolvimento são: as estenoses, que podem ocorrem na anastomose, na via venosa ou mesmo em veias centrais; a trombose; a presença de ramos colaterais e a insuficiência arterial ${ }^{11}$. Vários trabalhos vêm demonstrando a importância do reconhecimento e tratamento precoces dessas falhas, na tentativa de salvamento dos acessos ${ }^{10,12-15}$, por meio de procedimentos cirúrgicos e/ou endovasculares.

Este estudo teve como objetivo avaliar a perviedade precoce das FAV, definida como a patência da fístula nos primeiros 30 dias após a sua confecção ${ }^{16}$, e tentar identificar as possíveis causas para essa falha precoce.

\section{Material e métodos}

Foram estudados todos os pacientes encaminhados pelo Serviço de Nefrologia do Hospital do Servidor Público Municipal, diagnosticados com IRC e indicação de confecção de FAV para hemodiálise no período de agosto de 2008 a janeiro de 2009. Informações contidas no protocolo de FAV do Serviço de Cirurgia Vascular, sobre a história vascular prévia (uso de cateteres venosos ou outros acessos venosos prévios), causa da IRC, comorbidades (HAS, DM, 
arteriopatias etc.) e diálise prévia, foram obtidas antes do procedimento, e realizou-se exame físico vascular avaliando a qualidade da veia (presença de flebites, hematomas, veia visível ou palpável), da artéria (presença e qualidade do pulso) e perfusão do membro onde seriam confeccionadas as FAV. No intraoperatório, foram avaliadas a qualidade da veia, por meio de seu aspecto externo, e sua perviedade, por meio da progressão de sonda uretral de diâmetro 4 fr. e 6 fr. intraluminal e da artéria (aspecto externo, amplitude do pulso, presença de calcificação etc.) e, após a realização da anastomose, a presença e qualidade do frêmito e/ou pulso.

Esses pacientes foram re-examinados no $1^{\circ}, 10^{\circ}$ e $30^{\circ}$ pós-operatório (PO), sendo avaliado o aspecto da ferida operatória, a presença de frêmito/pulso no trajeto da FAV, presença de alterações que estivessem prejudicando a maturação da FAV (estenoses, ramos colaterais, hematomas, infecções) e a qualidade da diálise pelo acesso, se o paciente já estivesse em uso do mesmo. Considerou-se FAV pérvia na presença de frêmito em trajeto da mesma ou pulso $>3+/ 4+$. $\mathrm{Na}$ presença apenas de pulso, investigou-se o motivo para ausência de frêmito - estenose e/ou ramos colaterais.

Para o estudo estatístico, utilizou-se o teste exato de Fisher com p significativo, quando menor que 0,05.

Os pacientes receberam, leram e assinaram o termo de livre esclarecimento e consentimento e concordaram em participar da pesquisa. O estudo foi apreciado e autorizado pelo Comitê de Ética em Pesquisa do Hospital do Servidor Público de São Paulo (parecer no 174/2009).

\section{Resultados}

Foram realizadas 31 FAV em nosso serviço no período estudado, sendo 18 pacientes do sexo masculino e 13 do sexo feminino. A média de idade foi de 63,06 anos, variando de 19 a 85 anos. Dez pacientes já se encontravam em programa de hemodiálise, todos em uso de cateter venoso central. A hipertensão arterial foi a causa mais comum da IRC encontrada (Tabela 1). Apenas dois pacientes apresentavam o membro superior esquerdo dominante e somente um apresentava insuficiência do arco palmar à manobra de Allen. $\mathrm{O}$ pulso arterial (radial, ulnar e braquial) estava presente em

Tabela 1. Perfil dos pacientes estudados.

\begin{tabular}{lcc}
\hline & $\mathrm{n}$ & $\%$ - variação \\
\hline Pacientes & 31 & 100 \\
Masculino/feminino & $18 / 13$ & $58,06 / 41,94$ \\
Idade média (anos) & 63,06 & $19-85$ \\
IRC dialítica & 10 & 32,25 \\
HAS & 29 & 93,54 \\
DM & 14 & 45,16 \\
\hline
\end{tabular}

todos os pacientes. Doze pacientes apresentavam veia visível ao exame físico; 17 apresentavam veia palpável; em 1, a veia foi avaliada por Doppler e em 1, a veia não foi identificada.

Vinte e seis FAV foram distais (Figura 1), todas do tipo radiocefálica (11 à direita e 15 à esquerda); 4 foram proximais, das quais dois do tipo braquiocefálica, dois do tipo braquiobasílica superficializada (todas à esquerda); 1 FAV foi confeccionada com alça de PTFE fêmoro-femoral esquerda (Figura 2), em paciente com trombose da veia cava superior. Nenhuma FAV com prótese em membros superiores foi necessária.

No intraoperatório, quatro pacientes apresentavam veia de aspecto desfavorável (flebite, pequeno calibre, ausência de dilatação ou endurecimento da parede). Em sete casos, não foi possível a progressão de sonda 6 Fr por mais de $10 \mathrm{~cm}$, demonstrando estenoses no trajeto. $\mathrm{Na}$ avaliação arterial intraoperatória, sete artérias apresentavam calcificações.

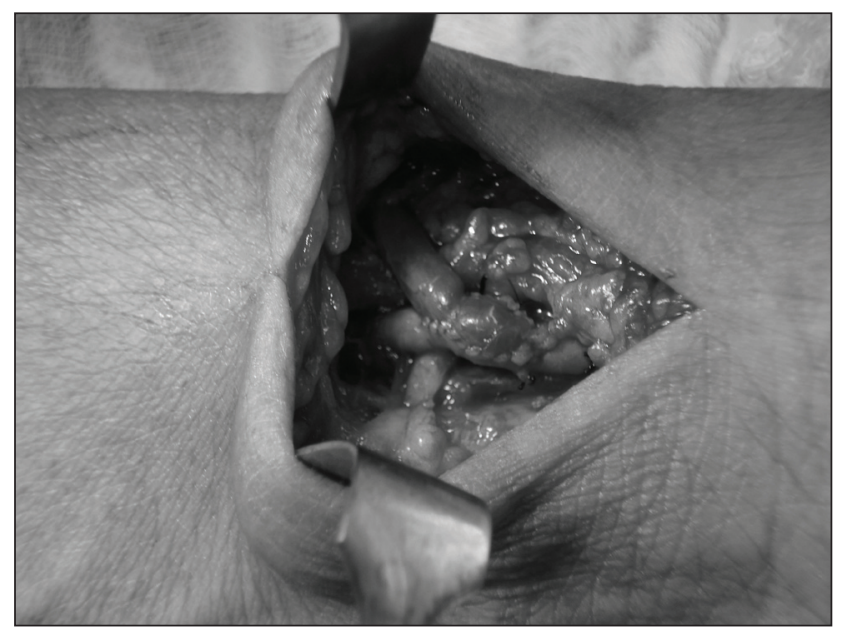

Figura 1. Aspecto intraoperatório; FAV rádio-cefálica confeccionada.

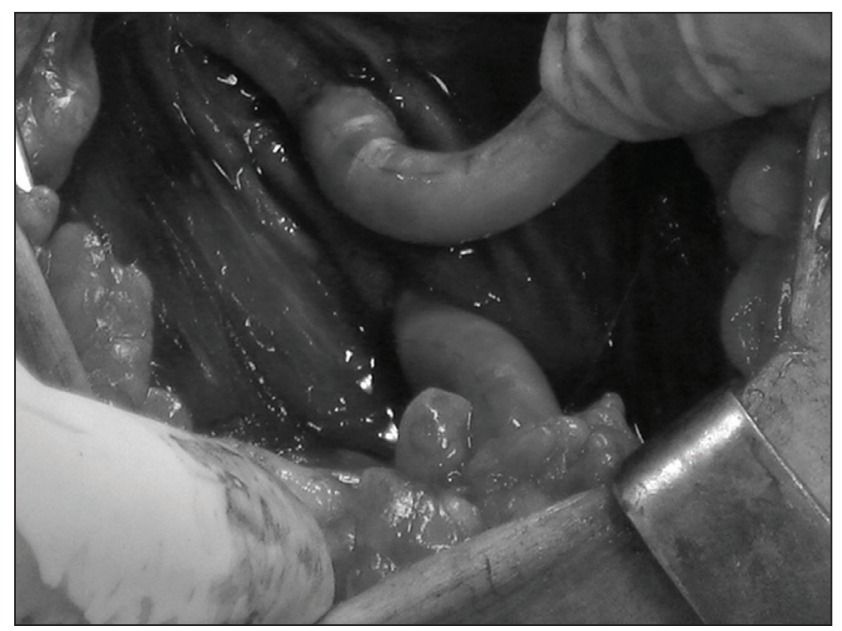

Figura 2. FAV fêmoro-femoral com prótese de PTFE. Acesso de exceção. 
Tabela 2. Fatores de risco presentes nos pacientes em que houve oclusão da FAV.

\begin{tabular}{lccc}
\hline & $\mathrm{n}$ & $\%$ & $\mathrm{p}$ \\
\hline Total de oclusões & 9 & - & - \\
Cateter central & $7 / 9$ & 77 & 0,01 \\
Veia não-visível & $2 / 9$ & 22 & 0,68 \\
Flebite da veia & $3 / 9$ & 33 & 0,06 \\
DM & $6 / 9$ & 66 & 0,18 \\
Progressão da Sonda 6Fr $<10 \mathrm{~cm}$ & $3 / 9$ & 33 & 0,42 \\
Calcificação arterial & $5 / 9$ & 55 & 0,08 \\
\hline
\end{tabular}

Vinte e dois pacientes receberam antibioticoprofilaxia com Cefazolina $1 \mathrm{~g}$ endovenosa no momento da anestesia, sendo distribuídos de maneira aleatória para o uso ou não desta. Os pacientes apresentavam frêmito na FAV ao término da cirurgia.

A taxa de perviedade no primeiro mês foi de $71 \%$ dos casos (22 pacientes). Das nove oclusões, 2 ocorreram no $1^{\circ}$ PO (as quais não foram reabordadas por aspecto ruim da veia no intraoperatório), 6 foram notadas no retorno do $10^{\circ}$ PO e 1 no retorno de 30 dias. Todas as oclusões ocorreram por trombose da FAV. Oito dos insucessos eram em FAV distais e um era proximal. $\mathrm{O}$ uso de cateteres venosos centrais no momento da confecção da FAV apresentou-se como fator de risco para sua oclusão $(\mathrm{p}=0,01)$. Apesar de não alcançar significância estatística neste estudo, a veia de aspecto desfavorável (flebites e de pequeno calibre) e as calcificações arteriais mostraram-se como fatores importantes para falência precoce da FAV (Tabela 2). O fato de a veia não ser visível ao exame físico, a não progressão da sonda 6 fr por mais de $10 \mathrm{~cm}$ na veia e o diabetes melito (DM) não se mostraram como fatores de risco para fechamento da FAV.

Seis pacientes apresentaram hematomas de ferida operatória (FO), todos tratados clinicamente e resolvidos com sucesso. Quatro pacientes apresentaram infecção da FO, todos leves (hiperemia de borda) e foram tratados com antibioticoterapia. A antibioticoprofilaxia não reduziu a taxa de infecção da FO neste estudo $(\mathrm{p}=0,22)$.

Dos 22 pacientes em que a FAV mantinha-se pérvia no retorno de 30 dias, apenas 5 já estavam em uso do acesso para hemodiálise, todos de forma adequada.

\section{Discussão}

As FAV continuam sendo o acesso vascular para hemodiálise mais aceito e seguro. Segundo recomendações do $\mathrm{K}^{-\mathrm{DOQI}^{6}}{ }^{6}$, ao menos $50 \%$ dos pacientes em programa de hemodiálise deveriam ter uma FAV como acesso vascular. O paciente em seu primeiro ano de hemodiálise em uso de cateter venoso custa, em média, 19.000 dólares a mais que um paciente com FAV, devido às maiores taxas de complicações ${ }^{6}$. Ortega et al. ${ }^{17}$ relatam maiores taxas de hospitalização e mortalidade nos pacientes em uso de cateteres em relação ao uso de FAV. Para O'Hare et al. ${ }^{18}$, a adequação do serviço e a experiência do cirurgião têm impacto positivo na escolha e confecção do acesso vascular.

A indicação precoce para confecção da FAV é de fundamental importância ${ }^{5}$. Como estas necessitam de um período para maturação, recomenda-se a cirurgia de maneira antecipada à hemodiálise, evitando o uso de cateteres e suas complicações. Como demonstrado neste estudo, a presença desses dispositivos também aumenta a chance de falência da FAV, sendo o paciente obrigado a se manter em uso do cateter. A hemodiálise também pode promover hipotensão, distúrbios de coagulação, entre outros, levando à oclusão precoce da FAV.

Neste estudo, a taxa de perviedade primária nos primeiros 30 dias foi do PO de $71 \%$, resultados semelhantes aos da literatura ${ }^{10}$. As características da veia (flebites e de pequeno calibre) e da artéria (calcificações) parecem os fatores mais importantes para o sucesso da confecção da FAV. Essas características, avaliadas habitualmente no exame físico, nem sempre correspondem aos achados intraoperatórios, o que aumenta a taxa de falência precoce. Uma alternativa de avaliação pré-operatória é a utilização da US Doppler. Robbin et al. ${ }^{8}$ referem melhora nas taxas de sucesso na confecção da FAV e queda de explorações cirúrgicas mal-sucedidas de 11 para $0 \%$. Os cuidados e orientações aos pacientes com IRC em fase inicial também são importantes, com intuito de preservar as artérias e veias comumente utilizadas na confecção da FAV 5 . Punções das veias cefálica e basílica devem ser evitadas, preservando-as. As veias do dorso da mão podem ser utilizadas para fins de coleta de exames e injeções de medicamento. Punções da artéria radial e braquial (para coleta de gasometria, por exemplo) também devem ser evitadas, podendo-se utilizar a artéria femoral ${ }^{5}$.

A perviedade ainda pode ser aumentada com o acompanhamento dos pacientes após a confecção da FAV. O reconhecimento precoce de estenoses, ramos colaterais e problemas no sistema arterial que mantém o acesso podem ser facilmente visualizados pelo exame físico ${ }^{11}$. Na suspeita clínica, os pacientes devem ser submetidos a um estudo contrastado da mesma e possível correção endovascular. Caso este não seja possível, a revisão cirúrgica deve ser programada $^{10,12,14}$.

Outra maneira de avaliação pós-operatória é a utilização da US Doppler. Com esta, é possível acompanhar o aumento tanto no diâmetro da veia como no fluxo, orientando o melhor momento para considerar as FAV maturadas e consequente liberação do uso do acesso para hemodiálise (Figura 3). Toregeani et al. ${ }^{9}$ referem, como critério de maturação, o 


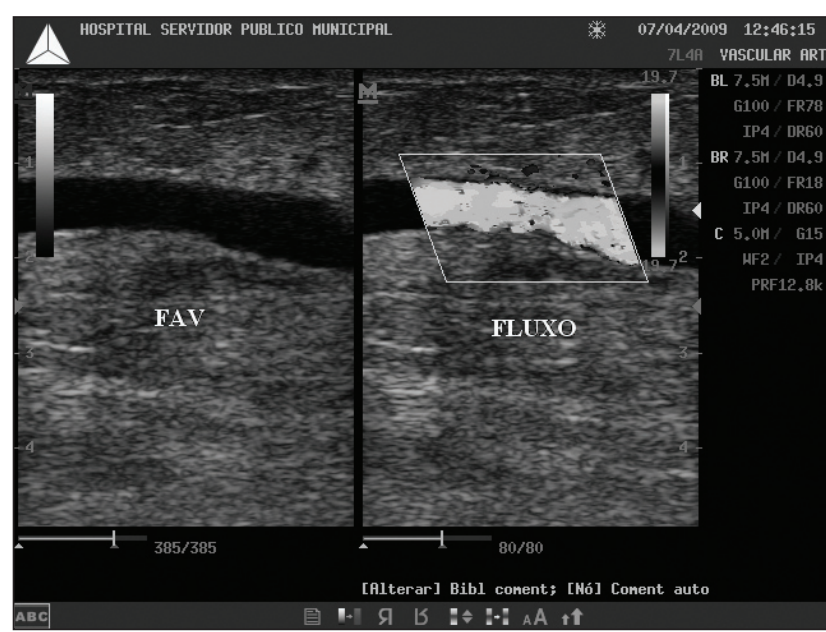

Figura 3. US Doppler mostrando FAV maturada com fluxo adequado.

diâmetro venoso maior que $4 \mathrm{~mm}$ e fluxo maior que $400 \mathrm{~mL} /$ minutos. Segundo o mesmo autor, esses parâmetros são geralmente alcançados por volta da quarta semana para FAV distais e da segunda semana para as FAV proximais.

A antiagregação plaquetária também vem sendo amplamente estudada como forma de prevenção de trombose precoce da FAV. Dember et al. ${ }^{19}$ relataram que o uso do Clopidogrel diminui a ocorrência da trombose do acesso, porém sem afetar sua maturação.

Com isso, conclui-se que a perviedade precoce neste estudo foi de $71 \%$. A utilização de cateteres para hemodiálise antes da confecção da FAV aumenta significativamente a chance da falência do acesso em 30 dias. Apesar de não alcançarem significância estatística, o aspecto desfavorável da veia e as calcificações arteriais parecem aumentar a taxa de oclusão precoce da FAV.

Deve-se considerar que, neste estudo, contou-se com uma pequena amostra. A não utilização do Doppler como rotina tanto pré como pós-operatória pode ter influenciado negativamente na perviedade das FAV, assim como a utilização prévia de cateteres venosos profundos em alguns casos.

\section{Referências}

1. DataSus. IDB 2006. Indicadores e dados básicos do Brasil. http:// www.datasus.gov.br/idb. 2006.Acessado em fevereiro de 2009.

2. Censo SBN 2008. http://sbn.org.br/censo.htm. 2008. Acessado em fevereiro de 2009.

3. Brescia MJ, et al. Chronic hemodialysis using venipuncture and a surgically created arteriovenous fistula. N Engl J Med. 1966;275:1089.

4. Hayashi R, Huang E, Nissenson A. Vascular Access for Hemodialysis. Natural Clinical Practice Nephrology. 2006;2(9):504-13.
5. Fistula First National Vascular Access Improvement Initiative. http://www.fistulafirst.org. 2009. Acessado em fevereiro de 2009.

6. NKF-K/DOQI. Clinical Practice Guidelines for Vascular Access: update 2006. Am J Kidney Dis. 2006;48(1):S177-247.

7. Sidawy AN, et al. The Society for Vascular Surgery: Clinical practice guidelines for the surgical placement and maintenance of arteriovenous hemodialysis access. J Vasc Surg. 2008;48:2S-25S.

8. Robbin ML, et al. US Vascular Mapping before Hemodialysis Access Placement. Radiology. 2000;217:83-8.

9. Toregeani JF, et al. Avaliação da maturação das fístulas arteriovenosas para hemodiálise pelo eco-Doppler colorido. J Vasc Bras. 2008;7(3):203-13.

10. Falk A. Maintenance and Salvage of arteriovenous fistula. J Vasc Interv Radiol. 2006;17:807-13.

11. Beathard GA. An Algorithm for the Physical Examination of Early Fistula Failure. Semin Dial. 2006;18:331-5.

12. Beathard GA, et al. Aggressive treatment of early fistula failure. Kidney International. 2003;64:1487-94.

13. Beathard GA. Successful Treatment of the Chronically Thrombosed Dialysis Access Graft: Resuscitation of Dead Grafts. Semin Dial. 2006;19:417-20.

14. Lipari G, et al. Outcomes of surgical revision of stenosed and thrombosed forearm arteriovenous fistula. Nephrol Dial Transplant. 2007;22:2605-12.

15. Ponikvar R. Surgical Salvage of Thrombosed Arteriovenous Fistulas and Grafts. Ther Apher Dial. 2005;9(3):245-9.

16. Sidawy AN, et al. Recommended standards for reports dealing with arteriovenous hemodialysis access. J Vasc Surg. 2002;35:603-10.

17. Ortega $T$, et al. The timely construction of arteriovenous fistulae: a key to reducing morbidity and mortality and to improving cost management. Nephrol Dial Transplant. 2005;20(3):598-603.

18. O'Hare AM, et al. Impact of surgeon and surgical center characteristics on choice of permanent vascular access. Kidney Int. 2003;64(2):681-9.

19. Dember LM, et al. Effect of Clopidogrel on Early Failure of Arteriovenous Fistulas for Hemodialysis: a randomized controlled trial. JAMA. 2008;299(18):2164-71.

Correspondência: Milton Alves das Neves Junior Rua Castro Alves, 60 - Secretaria do $5^{\circ}$ andar - Aclimação CEP: 01532-400 - São Paulo (SP) E-mail: miltonanj@yahoo.com.br

Contribuições dos autores Concepção e desenho do estudo: MANJ Análise e interpretação dos dados: MANJ, RCM, CCA, ARF, AP, MLSI, ER Coleta de dados: MANJ, RCM, CCA, ARF Redação do artigo: MANJ, RCM, CCA, ARF Revisão crítica do texto: AP, MLSI, ER Aprovação final do artigo*: MANJ, RCM, CCA, ARF, AP, MLSI, ER Análise estatística: MANJ Responsabilidade geral pelo estudo: MANJ * Todos os autores leram e aprovaram a versão final submetida ao I Vasc Bras 